\title{
Hierarchies and Uncertainty Measures on Pythagorean Fuzzy Approximation Spaces
}

\author{
Xiaojing LUO ${ }^{\text {a }}$, Jingjing SONG ${ }^{\text {a,b, }}$, Huili DOU ${ }^{a}$, Xibei YANG ${ }^{\text {a }}$, Taihua XU ${ }^{\text {a }}$ \\ ${ }^{a}$ School of Computer Science, Jiangsu University of Science and Technology, \\ Zhenjiang, Jiangsu 212100, P. R. China \\ ${ }^{\mathrm{b}}$ Key Laboratory of Data Science and Intelligence Application, Fujian Province \\ University, Zhangzhou, 363000, P. R. China
}

\begin{abstract}
In Granular Computing, the hierarchies and uncertainty measures are two important concepts to investigate the granular structures and uncertainty of approximation spaces. In this paper, hierarchies and uncertainty measures on pythagorean fuzzy approximation spaces will be researched. Firstly, the introduction and operations of pythagorean fuzzy granular structures are given, and three hierarchies and a lattice structure on pythagorean fuzzy approximation spaces are examined. The hierarchies are characterized by three order relations, the first order relation is defined on the inclusion relation of pythagorean fuzzy information granules, the second one is defined on the cardinality of pythagorean fuzzy information granules, and the third one is defined on the sum of the cardinality of pythagorean fuzzy information granules. The lattice structure is constructed on the first order relation on pythagorean fuzzy approximation spaces. Fuzzy information granularity and fuzzy information entropy are extended to describe the uncertainty of pythagorean fuzzy granular structures, and the relationship between the uncertainty measures and hierarchies are discussed. The examples show that hierarchies are effective to analyze the relationships among all granular structures on pythagorean fuzzy approximation spaces.
\end{abstract}

Keywords. Granular Computing, hierarchies, pythagorean fuzzy set, uncertainty measures

\section{Introduction}

Granular Computing(GrC), which was initiated by Zadeh [1,2], is an emerging computing paradigm of information processing. It is an effective way to simulate human being's thinking and divide the complex problem into a serious of relatively simple problems to facilitate the information processing. In $\mathrm{GrC}$, a collection of objects cluster with indivisibility, similarity, proximity, and functionality is called information granule [1]. All of these information granules together lead to an information granulation, which provide a visible underlying framework for problem solving. The information granulation of all objects in the universe results in a set of granules called a granular structure [1].

\footnotetext{
${ }^{1}$ Corresponding Author. E-mail: songjingjing108@163.com
} 
Different information granulations lead to different levels of abstraction and may present different granular views of our comprehension of a real world problem. $\mathrm{GrC}$ is searching for some suitable information granules so that a complex concept is able to approximate effectively at a specific level of granulation [1]. Up to now, $\mathrm{GrC}$ research is progressing rapidly $[3,4,5]$, the results show that $\mathrm{GrC}$ is becoming the mainstream of computer science.

After constructing all the granular structures on the approximation spaces, the hierarchies of the approximation spaces can be used to analyze the relationship among all the granular structures on approximate spaces [6]. Many scholars researched the hierarchies on various granular structures. For example, Yao [7] recommended to use hierarchical granulations to research of hierarchical rough set approximations. Zhang et al. [8] constructed the hierarchies of fuzzy quotient space by constructing the normalized isosceles distance function. Wang et al. [9] studied hierarchies knowledge space chain based on different knowledge granulation levels. In order to explore the finer or coarser relationships among multigranulation spaces, Yang et al. [10] have conducted three different hierarchies on the multigranulation rough set models. Song et al. [11] introduced knowledge distance $[12,13]$ to fuzzy environment, and proposed the local knowledge distance and global knowledge distance, such two knowledge distances are used to construct algebraic lattices, which are useful in characterizing the hierarchies on fuzzy information granulations. Huang et al. [14] investigated the hierarchies and a lattice structure of intuitionistic fuzzy set, and then further researched the uncertainty measures of intuitionistic fuzzy set. Some order relations were used to characterize the hierarchies on hesitant fuzzy approximation spaces by Tsang et al. [15].

In $\mathrm{GrC}$, a granular structure's uncertainty measure is a significant problem. Two main representations of uncertainty measure in different approximation spaces are information entropy and information granularity. An information granularity shows the discernibility of an information granule in a granular structure. If a granular structure is to be more recognizability, the smaller information granularity of granular structure is required [16]. Based on the different goals, the calculation of information granularity of granular structure is always a significant problem [17]. Granular structure entropy proposed by Shannon is an effective method to describe the information content of granular structure. Partition probability is used to define Shannon entropy, so the uncertainty of granular structure can not be described by Shannon entropy. Thus, the uncertainty of generalized granular structures are expressed by information entropy, which is obtained by extending Shannon entropy to different generalized granular structures $[18,19]$.

Fuzzy set, which was first put forward by Zadeh [20], deals with the uncertainty problems has achieved great success in many fields. Many different extend fuzzy set methods have been proposed by researchers to deal with more inaccurate information in practical applications, among which the intuitionistic fuzzy set proposed by Atanassov is one classic model [21]. Intuitionistic fuzzy set gives each element a membership degree and a nonmembership degree, and requests the sum of membership degree and nonmembership degree must less than or equal to 1 . It should be noted that sometimes the sum of membership degree and nonmembership degree is greater than 1, which can not be dealed with intuitionistic fuzzy set. To address this problem, Yager $[22,31,32,33]$ proposed pythagorean fuzzy set, which is obtained when the sum of squares of its membership degree and nonmembership degree is less than or equal to 1 . Therefore, pythagorean fuzzy set is one extension of fuzzy set, which is a direct promotion of intuitionistic fuzzy 
set. The pythagorean fuzzy set not only retains the advantages of the intuitionistic fuzzy set in processing uncertain information, but also broadens the application range of intuitionistic fuzzy set. Pythagorean fuzzy set has attracted by many scholars [26]- [30], but the hierarchy of pythagorean fuzzy approximation spaces has been rarely discussed, so the relationship among all the granular structures on pythagorean fuzzy approximation spaces can not be characterized.

Based on the descriptions above, there are three points to consider in GrC: information granulation, hierarchies and information granularity. Information granulation aims at granulating the objects in the universe of discourse into information granulation structure. Hierarchies with the purpose of sorting the information granulation structures on the approximation spaces. And the information granularity is used for measuring the granulation degree of an information granular structure [14]. As far as we know, there is no unified framework for these three developments, which is also important for the study of the pythagorean fuzzy granular structure. That is what drives our research. The motivation of this paper is to research the hierarchies and uncertainty measures of pythagorean fuzzy approximation spaces. To characterize the hierarchies on pythagorean fuzzy approximation spaces, three order relations on pythagorean fuzzy approximation spaces are proposed and some operations are defined on them, and then their lattice structures are given. Furthermore, the information granularity and information entropy to pythagorean fuzzy approximation spaces are extended to describe the uncertainty measures of pythagorean fuzzy approximation spaces. At last, the relationships between uncertainty measures and hierarchies of pythagorean fuzzy approximation spaces are discussed [14].

The rest of this study is organized as follows. Several basic notions are reviewed in Section 2 , such as the notions of fuzzy set, fuzzy granular structure and the order relations on fuzzy granular structures. On this basis, the pythagorean fuzzy set and pythagorean fuzzy approximation spaces are represented naturally. In Section 3, three order relations on pythagorean fuzzy approximation spaces are discussed. Furthermore, a lattice structure of pythagorean fuzzy approximation spaces, which based on the first order relation is investigated. The uncertainty measures of pythagorean fuzzy approximation spaces are discussed by using pythagorean fuzzy information granularity and pythagorean fuzzy information entropy in Section 4, and then the relationships among hierarchies and the uncertainty measures are established. The conclusions and the future perspectives of this study will be shown in Section 5 .

\section{Preliminary knowledge}

This section introduces some basic concepts of fuzzy set and three order relations of fuzzy information granulation, then the pythagorean fuzzy set and the pythagorean fuzzy approximation spaces are also introduced.

\subsection{Fuzzy Set}

Definition 1 [20] Let $U=\left\{x_{1}, x_{2}, \ldots, x_{n}\right\}$ be a nonempty set, which is called the universe of discourse. Assuming that $\hat{A}: U \rightarrow[0,1]$ such that $\mu_{\hat{A}}\left(x_{i}\right) \in[0,1]$ for each $x_{i} \in U$, then $\hat{A}=\left\{\frac{<\mu_{\hat{A}}\left(x_{i}\right)>}{x_{i}} \mid 1 \leq i \leq n\right\}$ is called a fuzzy set over $U$ where $\mu_{\hat{A}}\left(x_{i}\right)$ is the membership degree of element $x_{i}$. Furthermore, a fuzzy relation over $U$ is a fuzzy set such that $\hat{R}$ : $U \times U \rightarrow[0,1]$ 
Definition 2 [19] Let $U$ be the universe of discourse, a fuzzy granular structure over $U$ is defined as

$$
F(\hat{R})=\left(S_{\hat{R}}\left(x_{1}\right), S_{\hat{R}}\left(x_{2}\right), \cdots, S_{\hat{R}}\left(x_{n}\right)\right)
$$

where $S_{\hat{R}}\left(x_{i}\right)=\left\{\frac{r_{i j}}{x_{j}} \mid 1 \leq j \leq n\right\}(1 \leq i \leq n)$ is a fuzzy information granule induced by the element $x_{i}$ and the fuzzy relation $\hat{R}$.

The cardinality of $S_{\hat{R}}\left(x_{i}\right)$ is presented as $\left|S_{\hat{R}}\left(x_{i}\right)\right|=\sum_{j=1}^{n} r_{i j}(1 \leq i \leq n)$. According to Definition 2, the group of all fuzzy granular structures over $U$ is presented by $F(U, \hat{\Omega})$, which is denoted as a fuzzy approximation space, where $\hat{\Omega}$ represents the set of all fuzzy relations over $U$.

\subsection{Three Order Relations on Fuzzy Granular Structures}

Since order relation is a widely used method to characterize the hierarchies of granular structure, thus three order relations on fuzzy granular structures will be reviewed.

Definition 3 [19] Let $F(\hat{P}), F(\hat{Q}) \in F(U, \hat{\Omega}), \quad F(\hat{P})=\left(S_{\hat{P}}\left(x_{1}\right), S_{\hat{P}}\left(x_{2}\right), \cdots, S_{\hat{P}}\left(x_{n}\right)\right)$, $F(\hat{Q})=\left(S_{\hat{Q}}\left(x_{1}\right), S_{\hat{Q}}\left(x_{2}\right), \cdots, S_{\hat{Q}}\left(x_{n}\right)\right)$. The first order relation $\leq_{1}$ is defined as

$$
F(\hat{P}) \leq_{1} F(\hat{Q}) \Longleftrightarrow S_{\hat{P}}\left(x_{i}\right) \subseteq S_{\hat{Q}}\left(x_{i}\right)(1 \leq i \leq n)
$$

where $S_{\hat{P}}\left(x_{i}\right) \subseteq S_{\hat{Q}}\left(x_{i}\right)(1 \leq i \leq n)$ means that each membership of the elements in $S_{\hat{P}}$ is less than or equal to that in $S_{\hat{Q}}$, that is, $p_{i j} \leq q_{i j}(1 \leq i \leq n)$ for each element $x_{i}$.

Definition 4 [11] Let $F(\hat{P}), F(\hat{Q}) \in F(U, \hat{\Omega}), \quad F(\hat{P})=\left(S_{\hat{P}}\left(x_{1}\right), S_{\hat{P}}\left(x_{2}\right), \cdots, S_{\hat{P}}\left(x_{n}\right)\right)$, $F(\hat{Q})=\left(S_{\hat{Q}}\left(x_{1}\right), S_{\hat{Q}}\left(x_{2}\right), \cdots, S_{\hat{Q}}\left(x_{n}\right)\right)$. The second order relation $\leq_{2}$ is defined as

$$
F(\hat{P}) \leq_{2} F(\hat{Q}) \Longleftrightarrow\left|S_{\hat{P}}\left(x_{i}\right)\right| \leq\left|S_{\hat{Q}}\left(x_{i}\right)\right|(1 \leq i \leq n)
$$

Definition 5 [11] Let $F(\hat{P}), F(\hat{Q}) \in F(U, \hat{\Omega}), F(\hat{P})=\left(S_{\hat{P}}\left(x_{1}\right), S_{\hat{P}}\left(x_{2}\right), \cdots, S_{\hat{P}}\left(x_{n}\right)\right)$, $F(\hat{Q})=\left(S_{\hat{Q}}\left(x_{1}\right), S_{\hat{Q}}\left(x_{2}\right), \cdots, S_{\hat{Q}}\left(x_{n}\right)\right)$. The third order relation $\leq_{3}$ is defined as

$$
F(\hat{P}) \leq_{3} F(\hat{Q}) \Longleftrightarrow\left|\sum_{x_{i} \in U} S_{\hat{P}}\left(x_{i}\right)\right| \leq\left|\sum_{x_{i} \in U} S_{\hat{Q}}\left(x_{i}\right)\right|(1 \leq i \leq n)
$$

\subsection{Pythagorean Fuzzy Set}

Definition 6 [22] Let $U$ be the universe of discourse, a pythagorean fuzzy set $P$ in $U$ is denoted by $P=\left\{\frac{\left\langle\mu_{P}\left(x_{i}\right), v_{P}\left(x_{i}\right)>\right.}{x_{i}} \mid 1 \leq i \leq n\right\}$, where $\mu_{P}\left(x_{i}\right): U \rightarrow[0,1]$ is the degree of membership of element $x_{i}, v_{P}\left(x_{i}\right): U \rightarrow[0,1]$ is the degree of nonmembership of element $x_{i}$, and $0 \leq \mu_{P}\left(x_{i}\right)^{2}+v_{P}\left(x_{i}\right)^{2} \leq 1$. Furthermore, the hesitancy degree of $x_{i}$ to $P$ is denoted by $h_{P}\left(x_{i}\right)=\sqrt{1-\mu_{P}\left(x_{i}\right)^{2}-v_{P}\left(x_{i}\right)^{2}}$. 
For the sake of convenience, Zhang and Xu [25] called $\left(\mu_{P}\left(x_{i}\right), v_{P}\left(x_{i}\right)\right)$ a pythagorean fuzzy number denoted by $p=\left(\mu_{P}\left(x_{i}\right), v_{P}\left(x_{i}\right)\right)$.

Remark 1 Specially, if the membership degree $\mu_{P}\left(x_{i}\right)$ and nonmembership degree $\left.v_{P}\left(x_{i}\right)\right)$ of element $x_{i}$ in pythagorean fuzzy set $P$ satisfy the condition that $0 \leq \mu_{P}\left(x_{i}\right)+$ $\left.v_{P}\left(x_{i}\right)\right) \leq 1$, then the pythagorean fuzzy set $P$ is an intuitionistic fuzzy set [21]. The classical fuzzy set can be regarded as a special intuitionistic fuzzy set of the form $P=\left\{\frac{<\mu_{P}\left(x_{i}\right), 1-\mu_{P}\left(x_{i}\right)>}{x_{i}} \mid 1 \leq i \leq n\right\}$.

Definition 7 [23] Let $A=\left\{\frac{\left\langle\mu_{A}\left(x_{i}\right), v_{A}\left(x_{i}\right)>\right.}{x_{i}} \mid 1 \leq i \leq n\right\}$ and $B=\left\{\frac{\left\langle\mu_{B}\left(x_{i}\right), v_{B}\left(x_{i}\right)>\right.}{x_{i}} \mid 1 \leq i \leq n\right\}$ be two pythagorean fuzzy sets over the universe of discourse $U$, some operators, i.e., intersection $\cap$, union $\cup$, complement , equation $=$ on $A$ and $B$, and the inclusion relation between pythagorean fuzzy granular structures $A$ and $B$ can be defined as follows

1. $A \cap B=\left\{\frac{\leq \min \left\{\mu_{A}\left(x_{i}\right), \mu_{B}\left(x_{i}\right)\right\}, \max \left\{v_{A}\left(x_{i}\right), v_{B}\left(x_{i}\right)\right\}>}{x_{i}} \mid 1 \leq i \leq n\right\}$,

2. $A \cup B=\left\{\frac{<\max \left\{\mu_{A}\left(x_{i}\right), \mu_{B}\left(x_{i}\right)\right\}, \min \left\{v_{A}\left(x_{i}\right), v_{B}\left(x_{i}\right)\right\}>}{x_{i}} \mid 1 \leq i \leq n\right\}$,

3. $\left\langle A=\left\{\frac{\leq v_{A}\left(x_{i}\right), \mu_{A}\left(x_{i}\right)>}{x_{i}} \mid 1 \leq i \leq n\right\}\right.$ is the supplementary of $A$,

4. $A=B \Longleftrightarrow \mu_{A}\left(x_{i}\right)=\mu_{B}\left(x_{i}\right) \wedge v_{A}\left(x_{i}\right)=v_{B}\left(x_{i}\right)(1 \leq i \leq n)$,

5. $A \subseteq B \Longleftrightarrow \mu_{A}\left(x_{i}\right) \leq \mu_{B}\left(x_{i}\right) \wedge v_{A}\left(x_{i}\right) \geq v_{B}\left(x_{i}\right)(1 \leq i \leq n)$.

Let $U$ be the universe of discourse, the pythagorean fuzzy relation $R$ over $U$ can be represented by $n \times n$ matrix

$$
M_{R}=\left(\begin{array}{cccc}
\left\langle\mu_{R}\left(x_{1}, x_{1}\right), v_{R}\left(x_{1}, x_{1}\right)\right\rangle & \left\langle\mu_{R}\left(x_{1}, x_{2}\right), v_{R}\left(x_{1}, x_{2}\right)\right\rangle & \cdots & \left\langle\mu_{R}\left(x_{1}, x_{n}\right), v_{R}\left(x_{1}, x_{n}\right)\right\rangle \\
\left\langle\mu_{R}\left(x_{2}, x_{1}\right), v_{R}\left(x_{2}, x_{1}\right)\right\rangle & \left\langle\mu_{R}\left(x_{2}, x_{2}\right), v_{R}\left(x_{2}, x_{2}\right)\right\rangle & \cdots & \left\langle\mu_{R}\left(x_{2}, x_{n}\right), v_{R}\left(x_{2}, x_{n}\right)\right\rangle \\
\vdots & \vdots & \vdots & \vdots \\
\left\langle\mu_{R}\left(x_{n}, x_{1}\right), v_{R}\left(x_{n}, x_{1}\right)\right\rangle & \left\langle\mu_{R}\left(x_{n}, x_{2}\right), v_{R}\left(x_{n}, x_{2}\right)\right\rangle & \cdots & \left\langle\mu_{R}\left(x_{n}, x_{n}\right), v_{R}\left(x_{n}, x_{n}\right)\right\rangle
\end{array}\right),
$$

where $\mu_{R}\left(x_{i}, x_{j}\right), v_{R}\left(x_{i}, x_{j}\right) \in[0,1]$ and $0 \leq \mu_{R}^{2}\left(x_{i}, x_{j}\right)+v_{R}^{2}\left(x_{i}, x_{j}\right) \leq 1(1 \leq i, j \leq n)$.

We suppose that all pythagorean fuzzy relations in this paper are reflexive in the universe of discourse $U$, because reflexive is significant to characterize the similarity between objects, i.e., $\mu_{R}\left(x_{i}, x_{i}\right)=1, v_{R}\left(x_{i}, x_{i}\right)=0(1 \leq i \leq n)$ in $M_{R}$.

The group of pythagorean fuzzy relations over $U$ are denoted as $P F A S=(U, \Omega)$, which is called the pythagorean fuzzy approximation space over $U$, where $\Omega$ represents the set of pythagorean fuzzy relations over $U$.

\section{Hierarchies of Pythagorean Fuzzy Approximation Spaces}

Granular structure is a fundamental term in $\mathrm{GrC}$, and new granular structures can be generated by using several operators on granular structure. Four operators extended to fuzzy granular structures by Qian et al. [19]. In this section, the pythagorean granular structure is constructed and all objects are granulated into pythagorean fuzzy information granules. We also extend four operators of Qian on fuzzy granular space into pythagorean fuzzy granular space and then give three order relations on pythagorean fuzzy granular space, which constitute the hierarchies on pythagorean fuzzy approximation spaces. In the end, the lattice structure of the pythagorean approximation spaces is discussed . 


\subsection{Pythagorean Fuzzy Granular Structure}

Definition 8 A pythagorean fuzzy granular structure determined by pythagorean fuzzy relation $R$ in PFAS is defined as

$$
P(R)=\left(S_{R}\left(x_{1}\right), S_{R}\left(x_{2}\right), \cdots, S_{R}\left(x_{n}\right)\right)
$$

where $S_{R}\left(x_{i}\right)=\left\{\frac{\left.<r_{i j}^{+}, r_{i j}^{-}\right\rangle}{x_{j}} \mid 1 \leq j \leq n\right\}(1 \leq i \leq n), S_{R}\left(x_{i}\right)$ is the pythagorean fuzzy information granule induced by element $x_{i}$ and pythagorean fuzzy relation $R ; r_{i j}^{+}$is the similarity between elements $x_{i}$ and $x_{j}$, and $r_{i j}^{-}$is the dissimilarity between elements $x_{i}$ and $x_{j}$.

The collection of all pythagorean fuzzy granular structures over the universe $U$ can be represented by $\operatorname{PF}(U, \Omega)$. As is known to all, there is a 1-1 relationship between pythagorean fuzzy approximation space and pythagorean fuzzy granular structure space, thus, we do not distinguish the pythagorean fuzzy relation and pythagorean fuzzy granular structure between $(U, \Omega)$ and $P F(U, \Omega)$.

The fuzzy set's cardinality is important for investigating the information granulation and its measure of uncertainty, so is the pythagorean fuzzy set. The new cardinality of pythagorean fuzzy set is defined in Definition 9.

Definition 9 Let $P(R)=\left(S_{R}\left(x_{1}\right), S_{R}\left(x_{2}\right), \cdots, S_{R}\left(x_{n}\right)\right)$, where $S_{R}\left(x_{i}\right)=\left\{\frac{\left.<r_{i j}^{+}, r_{i j}^{-}\right\rangle}{x_{j}} \mid 1 \leq j \leq\right.$ $n\}(1 \leq i \leq n)$. The cardinality of $S_{R}\left(x_{i}\right)$ is regarded as

$$
\left|S_{R}\left(x_{i}\right)\right|=\frac{1}{2} \sum_{j=1}^{n}\left(1+\left(r_{i j}^{+}\right)^{2}-\left(r_{i j}^{-}\right)^{2}\right) .
$$

Accordingly, the cardinality of pythagorean fuzzy relation matrix is denoted by $\left|M_{R}\left(x_{i}\right)\right|=\frac{1}{2} \sum_{j=1}^{n}\left(1+\left(\mu_{R}\left(x_{i}, x_{j}\right)\right)^{2}-\left(v_{R}\left(x_{i}, x_{j}\right)\right)^{2}\right)$.

By employing this cardinality of pythagorean fuzzy information granule, we can construct the hierarchies and uncertainty measures on pythagorean fuzzy approximation spaces in this paper.

Definition 10 Let $P(L), P(Q) \in P F(U, \Omega)$, and $P(L)=\left(S_{L}\left(x_{1}\right), S_{L}\left(x_{2}\right), \cdots, S_{L}\left(x_{n}\right)\right), P(Q)=$ $\left(S_{Q}\left(x_{1}\right), S_{Q}\left(x_{2}\right), \cdots, S_{Q}\left(x_{n}\right)\right)$, where $S_{L}\left(x_{i}\right)=\left\{\frac{<l_{i j}^{+}, l_{i j}^{-}>}{x_{j}} \mid 1 \leq j \leq n\right\}(1 \leq i \leq n), S_{Q}\left(x_{i}\right)=$ $\left\{\frac{<q_{i j}^{+}, q_{i j}^{-}>}{x_{j}} \mid 1 \leq j \leq n\right\}(1 \leq i \leq n)$. Some operators, i.e., intersection $\cap$, union $\cup$, minus - , complement , equation $=$ and inequation $\neq$ on $P(L)$ and $P(Q)$ can be defined as follows

1. $P(L) \cap P(Q)=\left\{S_{L \cap Q}\left(x_{i}\right) \mid S_{L \cap Q}\left(x_{i}\right)=S_{L}\left(x_{i}\right) \cap S_{Q}\left(x_{i}\right), 1 \leq i \leq n\right\}$,

2. $P(L) \cup P(Q)=\left\{S_{L \cup Q}\left(x_{i}\right) \mid S_{L \cup Q}\left(x_{i}\right)=S_{L}\left(x_{i}\right) \cup S_{Q}\left(x_{i}\right), 1 \leq i \leq n\right\}$,

3. $P(L)-P(Q)=\left\{S_{L-Q}\left(x_{i}\right) \mid S_{L-Q}\left(x_{i}\right)=S_{L}\left(x_{i}\right) \cap\left\langle S_{Q}\left(x_{i}\right), 1 \leq i \leq n\right\}\right.$,

4. $\left\langle P(L)=\left\{2 S_{L}\left(x_{i}\right) \mid 1 \leq i \leq n\right\}\right.$,

5. $\left.P(L)=P(Q) \Longleftrightarrow S_{L}\left(x_{i}\right)=S_{Q}\left(x_{i}\right) \Longleftrightarrow l_{i j}^{+}=q_{i j}^{+} \wedge l_{i j}^{-}=q_{i j}^{-}, 1 \leq i, j \leq n\right\}$

6. $\left.P(L) \neq P(Q) \Longleftrightarrow S_{L}\left(x_{i}\right) \neq S_{\mathscr{L}}\left(x_{i}\right) \Longleftrightarrow l_{i j}^{+} \neq q_{i j}^{+} \wedge l_{i j}^{-} \neq q_{i j}^{-}, 1 \leq i, j \leq n\right\}$ 
where $S_{L \cap Q}=\left\{\frac{<\min \left\{l_{i j}^{+}, q_{i j}^{+}\right\}, \max \left\{l_{i j}^{-}, q_{i j}^{-}\right\}>}{x_{j}} \mid 1 \leq j \leq n\right\}, S_{L \cup Q}=\left\{\frac{<\max \left\{l_{i j}^{+}, q_{i j}^{+}\right\}, \min \left\{l_{i j}^{-}, q_{i j}^{-}\right\}>}{x_{j}} \mid 1 \leq\right.$ $j \leq n\},\left\langle S_{L}\left(x_{i}\right)=\left\{\frac{<l_{i j}^{-}, l_{i j}^{+}>}{x_{j}} \mid 1 \leq j \leq n\right.\right.$ and $\left.j \neq i\right\}(1 \leq i \leq n)$.

Proposition 1 Let $P(L), P(Q), P(R), P(\mathscr{U})$ and $P(\varphi) \in P F(U, \Omega), P(\mathscr{U})=\left(S_{\mathscr{U}}\left(x_{1}\right)\right.$, $\left.S_{\mathscr{U}}\left(x_{2}\right), \cdots, S_{\mathscr{U}}\left(x_{n}\right)\right)$, if $S_{\mathscr{U}}\left(x_{i}\right)=\left\{\frac{<1,0>}{x_{j}} \| 1 \leq i, j \leq n\right\}$, then $\mathscr{U}$ is the pythagorean fuzzy universal relation over $U, P(\mathscr{U})$ is the maximal pythagorean fuzzy granular structure; $P(\varphi)=\left(S_{\varphi}\left(x_{1}\right), S_{\varphi}\left(x_{2}\right), \cdots, S_{\varphi}\left(x_{n}\right)\right)$, if $S_{\varphi}\left(x_{i}\right)=\left\{\frac{<0,1>}{x_{j}} \mid 1 \leq j, i \leq n, j \neq i\right\}$, and $S_{\varphi}\left(x_{i}\right)=$ $\left\{\frac{\leq 1,0>}{x_{j}} \mid 1 \leq j=i \leq n\right\}$, then $\varphi$ is the pythagorean fuzzy identical relation over $U, P(\varphi)$ is the minimal pythagorean fuzzy granular structure. The operators, i.e., intersection $\cap$, union $\cup$, and complement ? on $P F(U, \Omega)$ satisfy the following properities.

1. Identity law: $P(L) \cap P(\mathscr{U})=P(L)$ and $P(L) \cup P(\varphi)=P(L)$.

2. Zero law: $P(L) \cup P(\mathscr{U})=P(\mathscr{U})$ and $P(L) \cap P(\varphi)=P(\varphi)$.

3. Commutative law: $P(L) \cap P(Q)=P(Q) \cap P(L), P(L) \cup P(Q)=P(Q) \cup P(L)$.

4. Association law: $(P(L) \cap P(Q)) \cap P(R)=P(L) \cap((P(Q) \cap P(R)), \quad(P(L) \cup$ $P(Q)) \cup P(R)=P(L) \cup((P(Q) \cup P(R))$.

5. Distributive law: $P(L) \cap(P(Q) \cup P(R))=(P(L) \cap(P(Q)) \cup(P(L) \cap P(R))$. $P(L) \cup(P(Q) \cap P(R))=(P(L) \cup P(Q)) \cap(P(L) \cup P(R))$.

6. Absorption law: $P(L) \cap(P(L) \cup P(Q))=P(L), P(L) \cup(P(L) \cap P(Q))=P(L)$.

7. De Morgan law: $(P(L) \cap P(Q))=\langle P(L) \cup\langle P(Q), \curlywedge(P(L) \cup P(Q))=\langle P(L) \cap$ $\langle P(Q)$.

\subsection{Three Order Relations on Pythagorean Fuzzy Approximation Spaces}

In this section, we give three order relations to characterize the hierarchies on different pythagorean fuzzy granular structures.

Definition 11 Let $P(L), P(Q) \in P F(U, \Omega), P(L)=\left(S_{L}\left(x_{1}\right), S_{L}\left(x_{2}\right), \cdots, S_{L}\left(x_{n}\right)\right), P(Q)=$ $\left(S_{Q}\left(x_{1}\right), S_{Q}\left(x_{2}\right), \cdots, S_{Q}\left(x_{n}\right)\right)$, the first order relation $\preceq_{1}$ is defined as follows

$P(L) \preceq_{1} P(Q) \Longleftrightarrow S_{L}\left(x_{i}\right) \subseteq S_{Q}\left(x_{i}\right)$ for any $1 \leq i \leq n$.

$P(L) \prec_{1} P(Q) \Longleftrightarrow P(L) \preceq{ }_{1} P(Q) \wedge P(L) \neq P(Q)$.

$P(L) \approx_{1} P(Q) \Longleftrightarrow S_{L}\left(x_{i}\right)=S_{Q}\left(x_{i}\right)$,

where $S_{L}\left(x_{i}\right) \subseteq S_{Q}\left(x_{i}\right)$ means that $\forall 1 \leq i, j \leq n$, we have $l_{i j}^{+} \leq q_{i j}^{+} \wedge l_{i j}^{-} \geq q_{i j}^{-}$.

Example 1 Let $U$ be the universe of discourse, $L, Q, S$ and $T$ are four pythagorean fuzzy relations over $U$, which are denoted by the following matrices, respectively.

$$
M_{L}=\left(\begin{array}{l}
<1.0,0.0><0.3,0.8><0.4,0.7><0.3,0.7><0.4,0.5> \\
<0.3,0.8><1.0,0.0><0.6,0.7><0.4,0.8><0.5,0.6> \\
<0.4,0.7><0.6,0.7><1.0,0.0><0.3,0.8><0.6,0.5> \\
<0.3,0.7><0.4,0.8><0.3,0.8><1.0,0.0><0.4,0.6> \\
<0.4,0.5><0.5,0.6><0.6,0.5><0.4,0.6><1.0,0.0>
\end{array}\right)
$$




$$
\begin{gathered}
M_{Q}=\left(\begin{array}{l}
<1.0,0.0><0.5,0.7><0.6,0.6><0.5,0.6><0.6,0.4> \\
<0.5,0.7><1.0,0.0><0.7,0.4><0.6,0.5><0.7,0.4> \\
<0.6,0.6><0.7,0.4><1.0,0.0><0.7,0.5><0.8,0.4> \\
<0.5,0.6><0.6,0.5><0.7,0.5><1.0,0.0><0.6,0.4> \\
<0.6,0.4><0.7,0.4><0.8,0.4><0.6,0.4><1.0,0.0>
\end{array}\right) \\
M_{S}=\left(\begin{array}{l}
<1.0,0.0><0.4,0.8><0.7,0.4><0.5,0.6><0.8,0.5> \\
<0.5,0.8><1.0,0.0><0.8,0.3><0.6,0.5><0.7,0.4> \\
<0.7,0.4><0.8,0.3><1.0,0.0><0.7,0.5><0.8,0.4> \\
<0.5,0.4><0.7,0.4><0.7,0.5><1.0,0.0><0.6,0.4> \\
<0.8,0.5><0.7,0.4><0.8,0.4><0.6,0.4><1.0,0.0>
\end{array}\right) \\
M_{T}=\left(\begin{array}{l}
<1.0,0.0><0.3,0.7><0.8,0.4><0.5,0.6><0.9,0.2> \\
<0.3,0.7><1.0,0.0><0.8,0.2><0.6,0.5><0.7,0.4> \\
<0.8,0.4><0.8,0.2><1.0,0.0><0.7,0.5><0.8,0.4> \\
<0.9,0.2><0.7,0.4><0.8,0.4><0.6,0.4><1.0,0.0>
\end{array}\right)
\end{gathered}
$$

We can observe that $\forall 1 \leq i, j \leq n$, we have $l_{i j}^{+}<q_{i j}^{+}$and $l_{i j}^{-}>q_{i j}^{-}$, which means that $S_{L}\left(x_{i}\right) \subseteq S_{Q}\left(x_{i}\right)$, thus $P(L) \prec_{1} P(Q)$ obtained. Then the pythagorean fuzzy granular structure $P(L)$ is finer than pythagorean fuzzy granular structure $P(Q)$, the order relation $\preceq_{1}$ can well differentiate the granularity between pythagorean fuzzy granular structures $P(L)$ and $P(Q)$.

We can observe that neither $S_{Q}\left(x_{1}\right) \subseteq S_{S}\left(x_{1}\right)$ nor $S_{S}\left(x_{1}\right) \subseteq S_{Q}\left(x_{1}\right)$ satisfies, which means the order relation $\preceq_{1}$ can not differentiate the granularity between the two pythagorean fuzzy granular structures $P(Q)$ and $P(S)$. To further distinguish their difference, a new finer criterion $\preceq_{2}$ will be proposed in Definition 12.

Definition 12 Let $P(L), P(Q) \in P F(U, \Omega), P(L)=\left(S_{L}\left(x_{1}\right), S_{L}\left(x_{2}\right), \cdots, S_{L}\left(x_{n}\right)\right), P(Q)=$ $\left(S_{Q}\left(x_{1}\right), S_{Q}\left(x_{2}\right), \cdots, S_{Q}\left(x_{n}\right)\right)$, the second order relation $\preceq_{2}$ is defined as follows

$P(L) \preceq_{2} P(Q) \Longleftrightarrow\left|S_{L}\left(x_{i}\right)\right| \leq\left|S_{Q}\left(x_{i}\right)\right|$ for any $1 \leq i \leq n$.

$P(L) \prec_{2} P(Q) \Longleftrightarrow P(L) \preceq_{2} P(Q) \wedge P(L) \neq P(Q)$.

$P(L) \approx_{2} P(Q) \Longleftrightarrow\left|S_{L}\left(x_{i}\right)\right|=\left|S_{Q}\left(x_{i}\right)\right|$ for any $1 \leq i \leq n$.

Continued from Example 1, we can easily verify that $\left|M_{Q}\left(x_{i}\right)\right|<\left|M_{S}\left(x_{i}\right)\right|$ for $i=1,2,3,4,5$, which means $\left|S_{Q}\left(x_{i}\right)\right|<\left|S_{S}\left(x_{i}\right)\right|$, thus $P(Q) \prec_{2} P(S)$ obtained. Then the pythagorean fuzzy granular structure $P(Q)$ is finer than pythagorean fuzzy granular structure $P(S)$, the order relation $\preceq_{2}$ can well differentiate the granularity between pythagorean fuzzy granular structures $P(Q)$ and $P(S)$.

By computing, we have $\left|M_{S}\left(x_{1}\right)\right|=3.065,\left|M_{S}\left(x_{4}\right)\right|=3.430,\left|M_{T}\left(x_{1}\right)\right|=3.370$, $\left|M_{T}\left(x_{4}\right)\right|=3.220$, then $\left|M_{S}\left(x_{1}\right)\right|<\left|M_{T}\left(x_{1}\right)\right|$ and $\left|M_{S}\left(x_{4}\right)\right|>\left|M_{T}\left(x_{4}\right)\right|$ exist, neither $\left|S_{S}\left(x_{i}\right)\right|<\left|S_{T}\left(x_{i}\right)\right|$ nor $\left|S_{T}\left(x_{i}\right)\right|<\left|S_{S}\left(x_{i}\right)\right|$ satisfies. Thus, the order relation $\preceq_{2}$ can not differentiate the granularity between the two granular structures $P(S)$ and $P(T)$. To further reveal their difference, a new finer criterion $\preceq_{3}$ will be introduced in Definition 13 . 
Definition 13 Let $P(L), P(Q) \in P F(U, \Omega), P(L)=\left(S_{L}\left(x_{1}\right), S_{L}\left(x_{2}\right), \cdots, S_{L}\left(x_{n}\right)\right), P(Q)=$ $\left(S_{Q}\left(x_{1}\right), S_{Q}\left(x_{2}\right), \cdots, S_{Q}\left(x_{n}\right)\right)$, the third order relation $\preceq_{3}$ is defined as follows

$$
\begin{aligned}
& P(L) \preceq_{3} P(Q) \Longleftrightarrow\left|\sum_{x_{i} \in U} S_{P}\left(x_{i}\right)\right| \leq\left|\sum_{x_{i} \in U} S_{Q}\left(x_{i}\right)\right|(1 \leq i \leq n) . \\
& P(L) \prec_{3} P(Q) \Longleftrightarrow P(L) \preceq_{3} P(Q) \wedge P(L) \neq P(Q) . \\
& P(L) \approx_{3} P(Q) \Longleftrightarrow\left|\sum_{x_{i} \in U} S_{P}\left(x_{i}\right)\right|=\left|\sum_{x_{i} \in U} S_{Q}\left(x_{i}\right)\right|(1 \leq i \leq n) .
\end{aligned}
$$

Continued from Example 1, by computing, we can verify that $\left|\sum_{x_{i} \in U} S_{S}\left(x_{i}\right)\right|=$ 17.295, $\left|\sum_{x_{i} \in U} S_{T}\left(x_{i}\right)\right|=17.700$, thus we have $\left|\sum_{x_{i} \in U} S_{S}\left(x_{i}\right)\right|<\left|\sum_{x_{i} \in U} S_{T}\left(x_{i}\right)\right|$ for $i=$ $1,2,3,4,5$, which implies $P(S) \prec_{3} P(T)$. Thus the pythagorean fuzzy granular structure $P(S)$ is finer than pythagorean fuzzy granular structure $P(T)$, the order relation ${ }_{3}$ can well differentiate the granularity between pythagorean fuzzy granular structures $P(S)$ and $P(T)$.

Proposition 2 The order relation $\preceq_{i}$ is a special case of the order relation $\preceq_{i+1}(i=$ $1,2)$.

The first order relation $\preceq_{1}$ is defined on the basis of pythagorean fuzzy inclusion relation between two pythagorean fuzzy granular structures, thus $\preceq_{1}$ is a partial order relation, however $\preceq_{2}, \preceq_{3}$ are not necessarily antisymmetric, thus they are quasi-order relations. The three order relations provide different criterias to discriminate the granularity of pythagorean fuzzy granular structures at multiple levels.

\subsection{Lattice Structure of Pythagorean Fuzzy Approximation Spaces}

Yang et al. [24] used a lattice structure from set distance to study the hierarchies of granular structures on a crisp approximate space. In this subsection, we will study the lattice structure of pythagorean fuzzy approximate spaces based on partial order relation $\preceq_{1}$.

Definition 14 Let $(L, \leq)$ be a poset, if there exist two operators $\wedge$ and $\vee$ on $L: L^{2} \rightarrow L$, $\forall a, b, c \in L$, such that

1. $a \wedge b=b \wedge a, a \vee b=b \vee a$;

2. $(a \wedge b) \wedge c=a \wedge(b \wedge c),(a \vee b) \vee c=a \vee(b \vee c)$;

3. $a \wedge b=b \Longleftrightarrow b \leq a, a \vee b=b \Longleftrightarrow a \leq b$.

We then call $(L, \leq)$ is a lattice.

Furthermore, we call $(L, \leq)$ a distributive lattice, if $a \wedge(b \vee c)=(a \wedge b) \vee(a \wedge c)$, $a \vee(b \wedge c)=(a \vee b) \wedge(a \vee c)$ is satisfied.

We call $(L, \leq)$ a complemented lattice, if $\forall a \in L$, there exists $a^{-1}$ such that $\left(a^{-1}\right)^{-1}=$ $a$ and $a \leq b \Longleftrightarrow b^{-1} \leq a^{-1}$. If there exist $0,1 \in L$ such that $0 \leq a \leq 1, \forall a \in L$, then we call 0 and 1 its minimal and maximal elements, respectively.

Theorem 1 Let $\cap, \cup$ and $\chi$ be defined on $P F(U, \Omega)$ as Definition 10 , then $\left(P F(U, \Omega), \preceq_{1}\right.$ $, \cap, \cup, 2)$ is a complemented distributive lattice.

Proof Based on Definitions 10, 14 and Proposition 1, it is straightforward.

In the complemented distributive lattice $\left(P F(U, \Omega), \preceq_{1}, \cap, \cup, 2\right), P(\varphi)$ and $P(\mathscr{U})$ defined in Proposition 1 are the minimal and maximal elements in $(P F(U, \Omega)$, respectively. 


\section{Uncertainty Measures for Pythagorean Fuzzy Approximation Spaces}

Two main uncertainty measures: granularity and information entropy, are important for an approximation space, In this section, we extend two measures, i.e., granularity and information entropy, to pythagorean fuzzy approximation spaces, and then analyze the relationships among the proposed order relationships and the two uncertainty measures.

\subsection{Granularity of Pythagorean Fuzzy Granular Structure}

Definition 15 [32] Let $F(\hat{R})=\left(S_{\hat{R}}\left(x_{1}\right), S_{\hat{R}}\left(x_{2}\right), \cdots, S_{\hat{R}}\left(x_{n}\right)\right)$ be a fuzzy granular structure, where $S_{\hat{R}}\left(x_{i}\right)=\left\{\frac{r_{i j}}{x_{j}} \mid 1 \leq j \leq n\right\}(1 \leq i \leq n)$. The granularity of $F(\hat{R})$ is defined as follows

$$
G F(\hat{R})=\frac{1}{n} \sum_{i=1}^{n} \frac{\left|S_{\hat{R}}\left(x_{i}\right)\right|}{n}=\frac{1}{n^{2}} \sum_{i=1}^{n} \sum_{j=1}^{n} r_{i j}
$$

Definition 16 Let $P(R) \in P F(U, \Omega)$, and $P(R)=\left(S_{R}\left(x_{1}\right), S_{R}\left(x_{2}\right), \cdots, S_{R}\left(x_{n}\right)\right)$, where $S_{R}\left(x_{i}\right)=\left\{\frac{\left.<r_{i j}^{+}, r_{i j}^{-}\right\rangle}{x_{j}} \mid 1 \leq j \leq n\right\}(1 \leq i \leq n)$. The granularity of pythagorean fuzzy granular structure $P(R)$ is defined as follows

$$
G P(R)=\frac{1}{n} \sum_{i=1}^{n} \frac{\left|S_{R}\left(x_{i}\right)\right|}{n}
$$

Theorem 2 Let $P(L), P(Q) \in P F(U, \Omega))$, then,

1. $P(L) \preceq{ }_{1} P(Q) \Longrightarrow G P(L) \leq G P(Q)$.

2. $P(L) \preceq_{2} P(Q) \Longrightarrow G P(L) \leq G P(Q)$.

3. $P(L) \preceq_{3} P(Q) \Longrightarrow G P(L) \leq G P(Q)$.

Proof Based on Definitions 11,12, 13 and Definition 16, it is obvious.

Example 2 Continued from Example 1.

The pythagorean fuzzy granularity of $P(L)$ is computed as follows

$\left|S_{P}\left(x_{1}\right)\right|=2.3150,\left|S_{P}\left(x_{2}\right)\right|=2.3650,\left|S_{P}\left(x_{3}\right)\right|=2.5500,\left|S_{P}\left(x_{4}\right)\right|=2.1850,\left|S_{P}\left(x_{5}\right)\right|=$ 2.8550, then $G P(L)=0.4906$.

The pythagorean fuzzy granularity of $P(Q)$ is computed as follows

$\left|S_{Q}\left(x_{1}\right)\right|=2.9250,\left|S_{Q}\left(x_{2}\right)\right|=3.2650,\left|S_{Q}\left(x_{3}\right)\right|=3.5250,\left|S_{Q}\left(x_{4}\right)\right|=3.2200,\left|S_{Q}\left(x_{5}\right)\right|=$ 3.6850, then $G P(Q)=0.6648$.

The pythagorean fuzzy granularity of $P(S)$ is computed as follows

$\left|S_{S}\left(x_{1}\right)\right|=3.0650,\left|S_{S}\left(x_{2}\right)\right|=3.3000,\left|S_{S}\left(x_{3}\right)\right|=3.8000,\left|S_{S}\left(x_{4}\right)\right|=3.4300,\left|S_{S}\left(x_{5}\right)\right|=$ 3.7000, then $G P(S)=0.6918$.

The pythagorean fuzzy granularity of $P(T)$ is computed as follows

$\left|S_{T}\left(x_{1}\right)\right|=3.3700,\left|S_{T}\left(x_{2}\right)\right|=3.3200,\left|S_{T}\left(x_{3}\right)\right|=3.9000,\left|S_{T}\left(x_{4}\right)\right|=3.2200,\left|S_{T}\left(x_{5}\right)\right|=$ 3.8900, then $G P(T)=0.7080$.

The results verify the Theorem 2 . 


\subsection{Information Entropy of Pythagorean Fuzzy Granular Structure}

Definition 17 [19] Let $F(\hat{P})=\left\{S_{\hat{P}}\left(x_{1}\right), S_{\hat{P}}\left(x_{2}\right), \cdots, S_{\hat{P}}\left(x_{n}\right)\right\}$ be a fuzzy granular structure. The fuzzy information entropy of $F(\hat{P})$ is defined as follows:

$$
E F(\hat{P})=\frac{1}{n} \sum_{i=1}^{n}\left(1-\frac{\left|S_{\hat{P}}\left(x_{i}\right)\right|}{n}\right)
$$

Definition 18 Let $P(R) \in P F(U, \Omega)$, and $P(R)=\left(S_{R}\left(x_{1}\right), S_{R}\left(x_{2}\right), \cdots, S_{R}\left(x_{n}\right)\right)$, the pythagorean fuzzy information entropy of $P(R)$ is defined as follows

$$
E P(R)=\frac{1}{n} \sum_{i=1}^{n}\left(1-\frac{\left|S_{R}\left(x_{i}\right)\right|}{n}\right)
$$

Theorem 3 Let $P(L), P(Q) \in P F(U, \Omega)$, then

1. $P(L) \preceq_{1} P(Q) \Longrightarrow E P(L) \geq E P(Q)$.

2. $P(L) \preceq_{2} P(Q) \Longrightarrow E P(L) \geq E P(Q)$.

3. $P(L) \preceq{ }_{3} P(Q) \Longrightarrow E P(L) \geq E P(Q)$.

Proof Based on Definitions 11, 12, 13 and Definition 18, it is obvious.

Example 3 Continue from Example 1.

By computing, $\left|S_{P}\left(x_{1}\right)\right|=2.3150,\left|S_{P}\left(x_{2}\right)\right|=2.3650,\left|S_{P}\left(x_{3}\right)\right|=2.5500,\left|S_{P}\left(x_{4}\right)\right|=$ $2.1850,\left|S_{P}\left(x_{5}\right)\right|=2.8550$ obtained, then the pythagorean fuzzy information entropy of $P(L)$ is $E P(L)=0.5094$.

By computing, $\left|S_{Q}\left(x_{1}\right)\right|=2.9250,\left|S_{Q}\left(x_{2}\right)\right|=3.2650,\left|S_{Q}\left(x_{3}\right)\right|=3.5250,\left|S_{Q}\left(x_{4}\right)\right|=$ $3.2200,\left|S_{Q}\left(x_{5}\right)\right|=3.6850$ obtained, then the pythagorean fuzzy information entropy of $P(Q)$ is $E P(Q)=0.3352$.

By computing, $\left|S_{S}\left(x_{1}\right)\right|=3.0650,\left|S_{S}\left(x_{2}\right)\right|=3.3000,\left|S_{S}\left(x_{3}\right)\right|=3.8000,\left|S_{S}\left(x_{4}\right)\right|=$ $3.4300,\left|S_{S}\left(x_{5}\right)\right|=3.7000$ obtained, then the pythagorean fuzzy information entropy of $P(S)$ is $E P(S)=0.3082$.

By computing, $\left|S_{T}\left(x_{1}\right)\right|=3.3700,\left|S_{T}\left(x_{2}\right)\right|=3.3200,\left|S_{T}\left(x_{3}\right)\right|=3.9000,\left|S_{T}\left(x_{4}\right)\right|=$ $3.2200,\left|S_{T}\left(x_{5}\right)\right|=3.8900$ obtained, then the pythagorean fuzzy information entropy of $P(T)$ is $\operatorname{EP}(T)=0.2920$.

The results verify the Theorem 3 .

\section{Conclusion}

Hierarchies and uncertainty measures are two key issues in the theory of GrC. The contributions in this paper are listed as follows

1. Three order relations on pythagorean fuzzy approximation spaces are discussed. On the basis of the first order relation, the lattice structure of pythagorean fuzzy approximation spaces is given.

2. The pythagorean fuzzy information granularity and pythagorean fuzzy information entropy are employed to characterize the uncertainty measures on pythagorean fuzzy approximation spaces. 
3. The relationships between the order relations and uncertainty measures are also discussed.

The followings topics deserve our further investigations.

1. We have only researched the hierarchies on pythagorean fuzzy approximation spaces by considering the degree of membership and nonmembership, did not take the hesitancy degree into account.

2. We have only characterized the uncertainty on pythagorean fuzzy approximation spaces with pythagorean fuzzy granularity and pythagorean fuzzy information entropy, some other uncertainty measures such as rough entropy and information Shannon entropy will be explored further.

3. This paper only mentioned the hierarchies and uncertainty measures of pythagorean fuzzy set, but does not apply it to specific practice, such as medical diagnosis, group decision making and other fields. We will continue to explore its applications in the future work.

\section{Acknowledgements}

This work was supported by the Natural Science Foundation of China (Nos. 61906078, 62076111, 62006099) and the Key Laboratory of Data Science and Intelligence Application, Fujian Province University (No. D1901).

\section{References}

[1] L. A. Zadeh. Towards a theory of fuzzy information granulation and its centrality in human reasoning and fuzzy logic. Fuzzy Sets and Systems, 1997, 19:111-127.

[2] L. A. Zadeh. Some reflections on soft computing, granular computing and their roles in the conception, design and utilization of information/intelligent systems. Soft Computing, 1998, 2:23-25.

[3] Xin Yang, Tianrui Li, Dun Liu, H. Fujita. A multilevel neighborhood sequential decision approach of three-way granular computing. Information Sciences, 2020, 538:119-141.

[4] Y. Y. Yao. Three-way granular computing, rough sets, and formal concept analysis. International Journal of Approximate Reasoning, 2020, 116:106-125.

[5] Shuyin Xia, Yunsheng Liu, Xin Ding, Guoyin Wang, Hong Yu, Yuoguo Luo. Granular ball computing classifiers for efficient, scalable and robust learning. Information Sciences, 2019, 483:136-152.

[6] P. Lingras, A. Elagamy, A. Ammar, Z. Elouedi. Iterative meta-clustering through granular hierarchy of supermarket customers and products. Information Sciences, 2014, 257:14-31.

[7] Y. Y. Yao. Information granulation and rough set approximation. International Journal of Intelligent Systems,2001, 16:87-104.

[8] Qinghua Zhang, Guoyin Wang, Xianquan Liu. Hierarchical structure analysis of fuzzy quotient space. Pattern Recognition and Artificial Intelligence, 2008, 5:627-634.

[9] Guoyin Wang, Qinghua Zhang. Uncertainty of rough sets in different knowledge granularities: uncertainty of rough sets in different knowledge granularities. Chinese Journal of Computers, 2009, 31:15881598.

[10] Xibei Yang, Yuhua Qian, Jingyu Yang. Hierarchical structures on multigranulation spaces. Journal of Computer Science and Technology, 2012, 27:1169-1183.

[11] Jingjing Song, Xibei Yang, Xiaoning Song, Hualong Yu, Jingyu Yang. Hierarchies on fuzzy information granulations: a knowledge distance based lattice approach. 2014, 27:1107-1117.

[12] Yuhua Qian, Jiye Liang, Chuangyin Dang. Knowledge structure, knowledge granulation and knowledge distance in a knowledge base. International Journal of Approximate Reasoning, 2009, 50:174-188. 
[13] Jiye Liang, Ru Li, Yuhua Qian. Distance: A more comprehensible perspective for measures in rough set theory. Knowledge-Based Systems, 2012, 27:126-136.

[14] Bing Huang, Chunxiang Guo, Huaxion Li, Guofu Feng, Xianzhong Zhou. Hierarchical structures and uncertainty measures for intuitionistic fuzzy approximation space. Information Sciences, 2016, 336:92114.

[15] Eric C. C. Tsang, Jingjing Song, Degang Chen, Xibei Yang. Order based hierarchies on hesitant fuzzy approximation space. International Journal of Machine Learning and Cybernetics, 2019, 10:1407-1422.

[16] Jianmin Ma, Wenxiu Zhang Yee Leung, Xiaoxue Song. Granular computing and dual Galois connection. Information Sciences, 2007, 177:5365-5377.

[17] Bingzhen Sun, Weimin Ma, Degang Chen. Rough approximation of a fuzzy concept on a hybrid attribute information system and its uncertainty measure. Information Sciences, 2014, 284:60-80.

[18] C. E. Shannon, A mathematical theory of communication. The Bell System Technical Journal, 1948, 27:379-423.

[19] Yuhua Qian, Jiye Liang, Weizhi Wu, Chuangyin Dang. Information granularity in fuzzy binary GrC model. IEEE Transactions on Fuzzy Systems, 2011, 19:253-264.

[20] L. A. Zadeh. Fuzzy sets. Information AND Control, 1965, 8:338-353.

[21] K. T. Atanassov. Intuitionistic fuzzy sets. Fuzzy Sets and Systems, 1986, 20:87-96.

[22] R. R. Yager. Pythagorean fuzzy subsets[C] 2013 Joint IFSA World Congress and NAFIPS Annual Meeting. Canada, 2013, 57-61.

[23] Xindong Peng, Huiyong Yuan, Yong Yang. Pythagorean fuzzy information measures and their applications. International Journal of Intelligent Systems, 2017, 32:991-1029.

[24] Xibei Yang, Yuhua Qian, Jingyu Yang. On characterizing hierarchies of granulation structures via distances. Fundamenta Informaticae, 2013, 123: 365-380.

[25] Xiaolu Zhang, Zeshui Xu. Extension of TOPSIS to multiple criteria decision making with pythagorean fuzzy sets. International Journal of Intelligent Systems, 2014, 29:1061-1078.

[26] Guangming Lang, Duoqian Miao, H. Fujita. Three-way group conflict analysis based on pythagorean fuzzy set theory. IEEE Transactions on Fuzzy Systems, 2020, 28:447-461.

[27] Anhui Tan, Suwei Shi, Weizhi Wu, Jinjin Li, W. Pedrycz. Granularity and entropy of intuitionistic fuzzy information and their applicatiions. IEEE Transactions on Cybernetics, DOI: 10.1109/TCYB.2020.2973379

[28] Decui Liang, Yinrunjie Zhang, Zeshui Xu, Adam Jamaldeen. Pythagorean fuzzy VIKOR approaches based on TODIM for evaluating internet banking website quality of Ghanaian banking industry. Applied Soft Computing, 2019, 78: 583-594.

[29] Fuyuan Xiao, Weiping Ding. Divergence measure of pythagorean fuzzy sets and its application in medical diagnosis. Applied Soft Computing, 2019, 79:254-267.

[30] Decui Liang, Zeshui Xu, Dun Liu, Yao Wu. Method for three-way decisions using ideal TOPSIS solutions at Pythagorean fuzzy information. Information Sciences, 2018, 435:282-295.

[31] R. R. Yager. Pythagorean Membership Grades in Multicriteria Decision Making. IEEE Transactions on Fuzzy Systems, 2014, 22(4):958-965.

[32] Qinghua Hu, Daren Yu, Zongxia Xie, Jinfu Liu. Fuzzy probabilistic approximation spaces and their information measures. IEEE Transactions on Fuzzy Systems, 2006, 14:191-201.

[33] Jiye Liang, Zhongzhi Shi, D.Y. Li, M. J. Wierman. The information entropy, rough entropy and knowledge graulation in incomplete information system. International Journal of General Systems, 2006, 35:641-654. 